\title{
Interval Representations
}

R.H.N. SANTIAGO, B.R.C. BEDREGAL ${ }^{1}$, Departamento de Informática e Matemática Aplicada - DIMAp, Universidade Federal do Rio Grande do Norte UFRN, Natal, RN, Brazil

B.M. ACIÓLY2, Universidade Estadual do Sudoeste da Bahia - UESB, Departamento de Ciências Exatas - DCE, Vitória da Conquista, BA, Brazil.

\begin{abstract}
This paper presents the concept of interval representation and shows some of its properties. The concept is often applied in interval mathematics and captures the essence of that theory; namely: Interval analysis is a language that designates computations with real numbers. The idea of interval objects as representation of real objects is defined and its relation with some aspects of interval analysis is showed. Some of these relations are concerned with the topological aspects of intervals (Scott topology).
\end{abstract}

\section{Introduction}

One of the ideas behind interval analysis is that it is a kind of language which designates real computations, in the sense that an interval $[a, b]$ represents any real number $r \in[a, b]^{3}$. The quality of this representation coded by interval width - i.e. $b-a$. Good interval functions $F: \mathbb{I}(\mathbb{R}) \rightarrow \mathbb{I}(\mathbb{R})$ used to designate real functions $f: \mathbb{R} \rightarrow \mathbb{R}$ are those which preserve the order of set inclusion and consequently the error quality. Those functions, $F: \mathbb{I}(\mathbb{R}) \rightarrow \mathbb{I}(\mathbb{R})$, have the following property:

$$
x \in[a, b] \Rightarrow f(x) \in F([a, b]) .
$$

This property capture the main requirement of interval analysis; the correctness of interval methods; namely it is enough to compute with intervals to obtain the resulting real number $f(x) \in F([a, b])$. This required property can be observed in Moore [5] theorem 3.1, p.21: "If $F$ is inclusion monotonic interval extension of $f$, then $\bar{f}\left(X_{1}, \ldots, X_{n}\right) \subseteq F\left(X_{1}, \ldots, X_{n}\right) " 4$.

In this paper we study functions which satisfies (1.1) and their relation with Scott continuity. We close the work with a brief result (Proposition 4.1) about the incompatibility between Scott/Moore continuity and computability.

\footnotetext{
${ }^{1}$ regivan,bedregal@dimap.ufrn.br. Both authors were partially supported by Project Ferus of International Cooperation CNPq-INRIA.

2 bma@uesb.br

${ }^{3} \mathrm{~A}$ concrete example of the field where interval computations are applied can be see in Loh and Walster [3].

${ }^{4}$ Where $\bar{f}(X)=f(X)=\{f(x): x \in X\}$.
} 


\section{Intervals}

A set $I \subseteq \mathbb{R}$ is an interval ${ }^{5}$ if whenever $x, y \in I$ and $x<y$, then every $z \in \mathbb{R}$ which satisfies $x \leq z \leq y$ also belongs to $I$. In what follows we show some required results on connectedness.

Lemma 2.1. A subset of $A \subseteq \mathbb{R}$ is connected iff $A$ is an interval.

Lemma 2.2. Continuous functions preserves connectedness; i.e. if $\left(A, \Omega_{1}\right)$ and $\left(B, \Omega_{2}\right)$ are topological spaces and $f: A \rightarrow B$ is a continuous function, then for all connected subset $C \subseteq A, f(C) \in \Omega_{2}$ is also connected (see [4]).

Straightforward from Lemmas 2.1 and 2.2 , we derive the following corollary.

Corollary 2.1. If $f: \mathbb{R} \rightarrow \mathbb{R}$ is continuous and $I \subseteq \mathbb{R}$ is an interval, then $f(I)$ is also an interval.

This corollary does not guarantee that any kind of interval is mapped on an interval of the same kind, e.g., it does not mean that $f([a, b])=[c, d]$. However, there are some conditions such that closed intervals are mapped to closed intervals by continuous functions. We trivially derive the following lemma.

Lemma 2.3. If $f: \mathbb{R} \rightarrow \mathbb{R}$ is continuous and $I \subseteq \mathbb{R}$ is a closed interval and there exist $x_{0}, x_{1} \in I$ such that for each $z \in I, f\left(x_{0}\right) \leq f(z) \leq f\left(x_{1}\right)$, then $f(I)$ is also a closed interval.

Proof. Since $x_{0}, x_{1} \in I$, then, trivially, $f\left(x_{0}\right), f\left(x_{1}\right) \in f(I)$ and by hypothesis for all $z \in I, f\left(x_{0}\right) \leq f(z) \leq f\left(x_{1}\right)$, so by corollary $2.1, f(I)$ is a closed interval.

From now on we use the word "interval" to designate just closed intervals, i.e., sets of the form $[a, b] \subseteq \mathbb{R}$.

\section{Aspects of Continuity}

\subsection{Metrics and Moore topology}

On the set of real numbers and on the set of intervals the notion of distance between two real numbers and two intervals, is given, respectively, by the functions: $d r(r, s)=|r-s|$ and $\operatorname{di}([a, b],[c, d])=\max (d r(a, c), d r(b, d))$. Those metrics are called euclidean metric and Moore metric[5], respectively. The pairs $(\mathbb{R}, d r)$ and $(\mathbb{I}(\mathbb{R}), d i)$ are called, respectively, the metric spaces of real numbers and Moore intervals. Given a metric space $(A, d)$ it is possible to define a topological space $(A, \Omega)$ induced by $d$, where the basic open sets are open balls $B(r, \epsilon)=\{s \in A: d(r, s)<\epsilon\}$. The metric of real numbers defines exactly the Euclidean topology, and the basic open sets can be open intervals $(r-\epsilon, r+\epsilon)$ for all $r \in \mathbb{Q}$. The set of degenerated intervals endowed with the metric $d i$ coincides with the topology of real numbers ${ }^{6}$.

\footnotetext{
${ }^{5}$ An interval can be a set of the form $(a, b),[a, b],(a, b],[a,+\infty)$, etc.

${ }^{6}$ Or in the topological language: They are homeomorphic spaces.
} 
A function $f: A \rightarrow B$, where $(A, d)$ and $\left(B, d^{\prime}\right)$ are metric spaces, is called continuous at $a \in A$ if, for every $\epsilon>0$, there is $\delta>0$, such that for every $x \in A$, if $d(x, a)<\delta$, then $d^{\prime}(f(x), f(a))<\epsilon . f$ is a continuous function if it is continuous in every $a \in A$. This notion of continuity coincides with the topological notion mentioned above.

\subsection{Scott-continuity and ord-continuity}

The theory of partially ordered sets has been used along the time for semantics of programming languages (see Stoy [8]). A partially ordered set is a pair $(A, \leq)$ where $\leq$ is a reflexive, transitive, and antisymmetric binary relation on $A$ called partial order on $A$. The ideas of information applied to the semantics of programming languages are modelled by partial orders; where the concepts of converging sequences can be extended by directed sets, $\Delta$, which are non-empty sets such that for every pair $x, y \in \Delta$, there is $z \in \Delta$ such that $x \leq z$ and $y \leq z$. The limits are modelled by supremums, $\bigsqcup \Delta$, which are defined by (1) $\bigsqcup \Delta$ is an upper bound of $\Delta^{7}$ and (2) for any upper bound $u$ of $\Delta, \sqcup \Delta \leq u$. A partially ordered set where every directed set has supremum is called directed complete partial order, or just dcpo, those partial orders abstract the well known property of real numbers which every convergent sequence has limit. The idea of continuous function on real numbers, where the limits of convergent sequences are preserved, is also extended to dcpos in the following sense: A function $f: A \rightarrow B$, where $A$ and $B$ are dcpos is an ord-continuous function if for every directed set $\Delta, f(\bigsqcup \Delta)=\bigsqcup f(\Delta)$. The monotonicity ${ }^{8}$ of any continuous function is trivially derived from ord-continuity.

In what follows we show how the idea of continuity on dcpos can be applied on the set of closed intervals, and how it is related with the standard notion of continuity which belongs to the field of topology.

In the set of closed intervals $\mathbb{I}(\mathbb{R})=\{[a, b]: a \in \mathbb{R}, b \in \mathbb{R}$ and $a \leq b\}$ the following partial order makes the pair $(\mathbb{I}(\mathbb{R}), \sqsubseteq)$ an $\omega$-continuous dcpo [7, 2]: Given $[a, b],[c, d] \in \mathbb{I}(\mathbb{R}),[a, b] \sqsubseteq[c, d]$ iff $a \leq c \leq d \leq b$. This dcpo is a richer structure ${ }^{9}$ such that its element can be viewed as partial information of real numbers. The intuition is that any interval $[a, b]$ is a partial information of a degenerated interval $^{10}[r, r]$, whenever $r \in[a, b]$, and $[r, r]$ only informs about itself. In the language of domain theory it means that a degenerated interval is a totally defined object. It is also possible to define a relation which captures the notion of approximation for intervals, in the sense that an interval $[a, b]$ is strongly below another interval $[c, d],[a, b] \ll[c, d]$, whenever $a<c \leq d<b$. This relation, which is called interval way-below associated with the order " $\sqsubseteq$ ", is such that it is possible to define a topology where the continuity of functions coincides with the preservation of the supremums. We will use $\uparrow[a, b]$, to denote the set $\{[c, d]:[a, b] \ll[c, d]\}$.

A monotonic function $F: \mathbb{I}(\mathbb{R}) \rightarrow \mathbb{I}(\mathbb{R})$ is a function such that if $[a, b] \sqsubseteq[c, d]$ then $F([a, b]) \sqsubseteq F([c, d])$. Interval monotonic functions have an important role with

\footnotetext{
${ }^{7} \sqcup \Delta \in A$ and for every $d \in \Delta, d \leq \bigsqcup \Delta$.

${ }^{8} \mathrm{~A}$ function $f: A \rightarrow B$, where $A$ and $B$ are partially ordered sets is monotonic if $x \leq y$ implies $f(x) \leq f(y)$.

${ }^{9}$ Called continuous Scott-domain. We will not show it in detail.

${ }^{10} \mathrm{An}$ interval with the same endpoints.
} 
respect to the preservation of the error estimative; namely since an interval can be used to represent a real number with an estimative of error (its width), the monotonicity guarantees that as shorter is the error of the input interval as shorter will be the error of the resulting interval.

On any dcpo $(A, \leq)$ it is possible to define a topology called Scott-topology, $\Omega_{S}(A)$ whose open sets have the following properties: For all $O \in \Omega_{S}(A)$, (1) if $x \in O$ and $x \leq y$, then $y \in O^{11}$, and (2) if $\Delta$ is a directed set and $\sqcup \Delta \in O$, then $\Delta \cap O \neq \emptyset$.

According to Acióly and Bedregal [1]:

"...the interval space with the Scott topology, $\left\langle\mathbb{I}(\mathbb{R}), \Omega_{S}(\mathbb{I}(\mathbb{R}))\right\rangle$, is a nice environment to do computation in the functional language sense. A denumerable basis for this topology is the set $\{\uparrow[p, q]:[p, q] \in \mathbb{I}(\mathbb{Q})\}$.

Any topological space endowed with a Scott topology gives rise to an information theory whose non-total elements are seen as partial objects. The order which is compatible with this topology is an information order that allows us to do qualitative comparisons between the elements of the space. Therefore in those spaces the mathematics developed are essentially qualitative, differently from that derived from a Hausdorff topology whose mathematics is mainly quantitative. This, however, does not mean that we cannot develop quantitative mathematics with dcpo's."

Lemma 3.1. If $D$ is a dcpo, then the set $U_{x}=\{z \in D: z \not \leq x\}^{12}$ is a Scott open set.

Lemma 3.2. Let $f: A \rightarrow B$ be a function, where $A$ and $B$ are dcpo's, if $f$ is continuous with respect to Scott topology (i.e. $f$ is Scott-continuous), then $f$ is monotonic.

Lemma 3.3. A function $f: A \rightarrow B$, where $A$ and $B$ are dcpo's, is ord-continuous iff it is Scott-continuous.

The proposition above tell us that the notion of topological continuity can be expressed in terms of order, i.e. in terms of mononoticity and the preservation the supremums. We close this section with the result that euclidean topology is extended to Scott-topology and any continuous function in the euclidean topology can be represented ${ }^{13}$ by a Scott-continuous function on $\mathbb{I}(\mathbb{R})$.

Lemma 3.4. The set $\uparrow \mathbb{I}(\mathbb{R})=\{\uparrow[a, b]:[a, b] \in \mathbb{I}(\mathbb{R})\}$ where $\uparrow[a, b]=\{[c, d]: a<$ $c \leq d<b\}$ is a basis of $\Omega_{S}(\mathbb{I}(\mathbb{R}))$.

Lemma 3.5. Let $\operatorname{Tot}(\mathbb{I}(\mathbb{R}))$ be the set of degenerated intervals, $\Omega_{S}(\operatorname{Tot}(\mathbb{I}(\mathbb{R})))$ the relative topology of $\Omega_{S}(\mathbb{I}(\mathbb{R}))$ restricted to $\operatorname{Tot}(\mathbb{I}(\mathbb{R}))$. Then, the set $B=\{([x, x],[y, y])$ : $x, y \in \mathbb{R}$ and $x<y\}$, where $([x, x],[y, y])=\{[z, z]: x<z<y\}$, is a base for $\Omega_{S}(\operatorname{Tot}(\mathbb{I}(\mathbb{R})))$.

\footnotetext{
11 i.e. $O$ is upper closed.

12 i.e. the collection of all points that either lie above $x$ or is incomparable with $x$.

${ }^{13}$ This notion of interval representation is informal for a while, but it will be formalized in the sequel.
} 
Theorem 3.1. Let $\operatorname{Tot}(\mathbb{I}(\mathbb{R}))$ be the set of degenerated intervals, $\Omega_{S}(\operatorname{Tot}(\mathbb{I}(\mathbb{R})))$ the relative topology of $\Omega_{S}(\mathbb{I}(\mathbb{R}))$ restricted to $\operatorname{Tot}(\mathbb{I}(\mathbb{R}))$, and $\mathcal{U}$ the usual Euclidean topology on $\mathbb{R}$. Then, there is an homeomorphism $f: \operatorname{Tot}(\mathbb{I}(\mathbb{R})) \rightarrow \mathbb{R}$.

Proof. Let $f: \operatorname{Tot}(\mathbb{I}(\mathbb{R})) \rightarrow \mathbb{R}$ be defined by $f([x, x])=x$. If $O \in \Omega_{S}(\operatorname{Tot}(\mathbb{I}(\mathbb{R})))$ then, by lemma $3.5, O=\bigcup_{i}\left(\left[x_{i}, x_{i}\right],\left[y_{i}, y_{i}\right]\right)$. So, $f(O)=\{f([z, z]):[z, z] \in$ $\left.\left(\left[x_{i}, x_{i}\right],\left[y_{i}, y_{i}\right]\right) \subseteq O\right\}=\bigcup_{i}\left(x_{i}, y_{i}\right) \in \mathcal{U}$. Trivially, $f^{-1}: \mathbb{R} \rightarrow \operatorname{Tot}(\mathbb{I}(\mathbb{R}))$ is defined by $f^{-1}(x)=[x, x]$. If $O \in \mathcal{U}$ then $O=\bigcup_{i}\left(x_{i}, y_{i}\right)$. So, $f^{-1}(O)=\left\{f^{-1}(z): z \in\right.$ $\left.\left(x_{i}, y_{i}\right) \subseteq O\right\}=\bigcup_{i}\left(\left[x_{i}, x_{i}\right],\left[y_{i}, y_{i}\right]\right) \in \Omega_{S}(\operatorname{Tot}(\mathbb{I}(\mathbb{R})))$. So, $f$ and $f^{-1}$ are continuous. Since $f \circ f^{-1}=i d$ and $f^{-1} \circ f=i d$ then $f$ is bijective. Therefore, $f^{-1}$ is continuous. Thus, $f$ is an homeomorphism.

\section{Interval extentions vs. interval representations}

Moore arithmetics brings to intervals some of the properties of real arithmetic, mainly the continuity of the operations. In other words, the continuity of real arithmetics is preserved and "hidden" in interval arithmetic. However, the extension of real functions to interval functions ${ }^{14}$ does not guarantee that continuity is preserved. For example, let $f: \mathbb{R} \rightarrow \mathbb{R}$ be the constant function $f(x)=5$, the function

$$
F([a, b])= \begin{cases}{[5,5],} & \text { if } a=b, \\ {[0,1],} & \text { otherwise }\end{cases}
$$

is trivially an extension of $f$, but it is not Moore-continuous, since given the basic open set $B([5,5], 1)=\{[a, b] \in \mathbb{I}(\mathbb{R}): d([a, b],[5,5])<1\}, F^{-1}(B([5,5], 1))=$ $\{[x, y] \in \mathbb{I}(\mathbb{R}): x=y\}$, is not an open set, otherwise it would be the union of basic open sets $B([r, s], \epsilon)$, for every real number $\epsilon>0$. However, no basic open set is contained in it. For example, clearly $\left[r, s+\frac{\epsilon}{2}\right] \in B([r, s], \epsilon)$ but $B([r, s], \epsilon)$ is not a subset of $F^{-1}(B([5,5], 1))=\{[x, y] \in \mathbb{I}(\mathbb{R}): x=y\}$. It is not also Scott-continuous, because it is not monotonic with respect to the information order.

There are interval functions which extend real functions, but with a lost of information, for example: The function $F[a, b]=[\operatorname{mid}([a, b]), \operatorname{mid}([a, b])]^{15}$ extends the identity function $\operatorname{Id}(x)=x$. However it would be expected that for all $x \in[a, b]$, $f(x) \in F([a, b])$, which is not true. Therefore it is important that if an interval $A$ represents a real numbers $r$ and a real function maps $r$ into $s$, then the interval function used to represent $f$, should map $A$ into $B$ such that $s \in B$. This intuition induces the notion of interval representation, which will mean that an interval object (an interval or an interval function) can be used to represent a real object (a real number or a real function). In the sequel, we propose a canonical interval representation of a real function $f, C I R(f)$, which always returns the best interval containing the image of $f$.

\footnotetext{
${ }^{14} \mathrm{~A}$ function $F: \mathbb{I}(\mathbb{R}) \rightarrow \mathbb{I}(\mathbb{R})$ is an extension of a function $f: \mathbb{R} \rightarrow \mathbb{R}$ if for all $x \in \mathbb{R}$ $F([x, x])=[f(x), f(x)][5]$ p. 21

${ }^{15} \operatorname{mid}([a, b])=\frac{b+a}{2}$, is called the midpoint of $[a, b]$.
} 
Definition 4.1. An interval $A$ represents every $a \in A$. Given the functions $f: \mathbb{R} \rightarrow \mathbb{R}$ and $F: \mathbb{I}(\mathbb{R}) \rightarrow \mathbb{I}(\mathbb{R}), F$ is an interval representation for $f$ if for all $A \in \mathbb{I}(\mathbb{R})$ and $a \in A, F(A)$ represents $f(a)$.

Therefore, at least, when we use interval mathematics to compute numerical functions, it is required to use functions which are interval representations. In what follows we show some properties of those functions.

Lemma 4.1. Some interval function $F: \mathbb{I}(\mathbb{R}) \rightarrow \mathbb{I}(\mathbb{R})$ does not represent any real function $f$.

Proof. Consider the following interval function:

$$
F([a, b])= \begin{cases}{[5,5],} & \text { if } a=b, \\ {[0,1],} & \text { otherwise }\end{cases}
$$

Suppose that $F$ represents a function $f$, then since $3 \in[2,4]$, then $f(3) \in$ $F([2,4])$ and $f(3) \in F([3,3])$. But $F([2,4])=[0,1]$ and $F([3,3])=[5,5]$, and so $f$ is not a function, what is a contradiction.

Lemma 4.2. Not every real function $f$ admits interval representations.

Proof. Given the function

$$
f(x)= \begin{cases}\frac{1}{x}, & \text { if } x>0 \\ 1, & \text { otherwise }\end{cases}
$$

there is no interval function $F$ such that for all $x \in[-1,1], f(x) \in F[-1,1]$, since $f$ is asymptotic at point 0 .

Observe, however, that if we consider partial interval functions it is still possible to define the notion of interval representation on the set of intervals $\{[a, b]: b \leq$ $0 \vee a>0\}$. Note also that only asymptotic functions $f: \mathbb{R} \rightarrow \mathbb{R}$ in some point $x$ bring this problem on. In other words, asymptotic real functions will obligate partiality on interval representations. For a while, partial interval representations theory will not be developed in this paper, instead we will consider only total nonasymptotic real functions and its interval representations. Some of the following propositions also hold, vacuously, for total asymptotic real functions.

Lemma 4.3. Every monotonic function $F: \mathbb{I}(\mathbb{R}) \rightarrow \mathbb{I}(\mathbb{R})$ represents some real function $f$.

Proof. Let $F: \mathbb{I}(\mathbb{R}) \rightarrow \mathbb{I}(\mathbb{R})$ be a monotonic function, with respect to the information order. Define $f: \mathbb{R} \rightarrow \mathbb{R}$ by $f(x)=\operatorname{mid}(F[x, x])$. Suppose that $x \in[a, b]$, then since $F$ is monotonic, $F[a, b] \sqsubseteq F[x, x]$, but $\operatorname{mid}(F[x, x])=f(x) \in F[x, x]$. So, $f(x) \in F[a, b]$, therefore $F$ represents $f$.

In some sense, this proposition reflects the fact that Moore [5] emphasizes the importance of monotonicity for interval functions. 
Corollary 4.2. Every Scott-continuous function $F: \mathbb{I}(\mathbb{R}) \rightarrow \mathbb{I}(\mathbb{R})$ represents some real function $f$.

Lemma 4.4. Not every interval representation is a monotonic function, and therefore an ord-continuous function.

Proof. Take for, example, the constant $f(x)=5$; the representation $F(A)=[4,6]$ if $[2,3] \sqsubseteq A$ or $F(A)=[5,5]$ otherwise. Observe that $[1,4] \sqsubseteq[2.5,2.6]$ but $F[1,4]=$ $[5,5] \nsubseteq[4,6]=F[2.5,2.6]$.

Corollary 4.3. The set of interval representations is a proper superset of interval monotonic functions.

The following results show that for every continuous function in $\mathbb{R}$, there exist an optimum interval representation for that; namely $C I R(f)$.

Theorem 4.1. Let $f: \mathbb{R} \rightarrow \mathbb{R}$ be a real function. If $f$ is a total non-asymptotic real function, then the interval function:

$$
C I R(f)([a, b])=[\min f([a, b]), \max f([a, b])]
$$

is well defined and it is an interval representation called canonical interval representation for $f$.

Proof. Straightforward.

Lemma 4.5. If $f: \mathbb{R} \rightarrow \mathbb{R}$ is continuous ${ }^{16}$ then for every $[a, b] \in \mathbb{I}(\mathbb{R}), C I R(f)([a, b])$ $=f([a, b])$.

Proof. Suppose that $f$ is continuous, given $[a, b] \in \mathbb{I}(\mathbb{R})$, by definition $\min (f[a, b]), \max (f[a, b]) \in C I R(f)([a, b]) \cap f([a, b])$, therefore there are $x_{0}, x_{1} \in$ $[a, b]$, such that $f\left(x_{0}\right)=\min (f[a, b])$ and $f\left(x_{1}\right)=\max (f[a, b])$, thus $f\left(x_{0}\right) \leq f\left(x_{1}\right)$ and for every $z \in[a, b], f\left(x_{0}\right) \leq f(z) \leq f\left(x_{1}\right)$. So by lemma 2.3, $f(I)$ is a closed interval. Clearly, the extreme of $f(I)$ are, respectively, $f\left(x_{0}\right)$ and $f\left(x_{1}\right)$, so $f(I)=\left[f\left(x_{0}\right), f\left(x_{1}\right)\right]=[\min (f[a, b]), \max (f[a, b])]=C I R(f)([a, b])$.

Corollary 4.4. If $f$ is continuous and $y \in C I R(f)([a, b])=f([a, b])$, then $f^{-1}(y) \cap$ $[a, b] \neq \emptyset$.

Note that if $f$ is an asymptotic real function at $x$, then for every $[a, b]$, where $x \in[a, b]$, there does not exist $[c, d]$ such that $f(x) \in[c, d]$, and therefore $f$ has no interval representation. Thus, if $f$ admits an interval representation, then $C I R(f)$ exists.

Theorem 4.2. For every interval representation $F$ of a real function $f, F \sqsubseteq$ $C I R(f)^{17}$.

\footnotetext{
${ }^{16}$ In the usual sense - according to euclidean topology.

${ }^{17}$ i.e., for every interval $A, F(A) \sqsubseteq C I R(f)(A)$.
} 
Proof. Suppose that $F$ is an interval representation for $f$, the for all $x \in[a, b]$, $f(x) \in F[a, b]$, so $f[a, b] \subseteq F[a, b]$, by theorem 4.5, CIR(f) $[a, b] \subseteq F[a, b]$, and then $F[a, b] \sqsubseteq C I R(f)[a, b]$

Therefore $C I R(f)$ is the best representation of a continuous function $f$. The next theorem, tell us in some sense that the continuity of $f$ induces the Scott-continuity in $\mathbb{I}(\mathbb{R})$.

Theorem 4.3. For every real continuous function $f, C I R(f)$ is Scott-continuous.

Proof. Let $f$ be a continuous function. According to lemma 3.3 it is enough to prove that $C I R(f)$ is ord-continuous. (1) $[a, b] \sqsubseteq[c, d]$ then $[c, d] \subseteq[a, b]$, so $f([c, d]) \subseteq$ $f([a, b])$, therefore $\min (f[a, b]) \leq \min (f([c, d])$ and $\max (f[a, b]) \geq \max (f([c, d])$, hence $C I R(f)([a, b]) \sqsubseteq C I R(f)([c, d])$. (2) Since $C I R(f)$ is monotonic then for every directed set $\Delta, C I R(f)(\Delta)$ is also directed and $C I R(f)(\sqcup \Delta)$ is one of its upper bounds, and then $\bigsqcup C I R(f)(\Delta) \sqsubseteq C I R(f)(\bigsqcup \Delta)$. So it is enough to prove that $C I R(f)(\bigsqcup \Delta) \sqsubseteq \bigsqcup C I R(f)(\Delta)$. If $y \in \bigsqcup C I R(f)(\Delta)$, then for every $f([a, b]) \in$ $C I R(f)(\Delta), f^{-1}(y) \cap[a, b] \neq \emptyset$. We need to prove that $\cap \Delta \cap f^{-1}(y) \neq \emptyset 18$. Suppose that $f^{-1}(y) \cap[a, b]=\emptyset$, then there exist $[a, b],[c, d] \in \Delta$ such that $f^{-1}(y) \cap$ $[a, b] \cap[c, d]=\emptyset$, because $\Delta$ is directed there exists $[e, f] \in \Delta$ where $[a, b],[c, d] \sqsubseteq$ $[e, f]$, but clearly $[e, f] \subseteq[a, b] \cap[c, d]$, and hence $f^{-1}(y) \cap[e, f]=\emptyset$, since $f$ is continuous, according to corollary 4.4 , for each $[a, b] \in \Delta, f^{-1}(y) \cap[a, b] \neq \emptyset$, which is a contradiction. So there exist $x \in \sqcup \Delta$ such that $f(x)=y$, and therefore $y \in C I R(f)(\bigsqcup \Delta)$.

Corollary 4.5. CIR is an operator which maps the set of real Euclidean continuous functions into the set of Scott-continuous function.

Proof. Observe that $C I R$ maps a continuous function in a unique ord-continuous function

Lemma 4.6. There exist non-continuous functions $f: \mathbb{R} \rightarrow \mathbb{R}$, such that $C I R(f)$ is monotonic.

Proof. We will prove that trunc is a counter-example. (1) trunc is not continuous: Consider the open ball $O=(4.5,5.5)$, then $\operatorname{trunc}^{-1}(O)=[5,6)$, which is not an open set. (2) $C I R(\operatorname{trunc})[a, b]=[\min \{\operatorname{trunc}(x . y): a \leq x . y \leq b\}, \max \{\operatorname{trunc}(x . y): a \leq$ $x . y \leq b\}]=[\min \{\operatorname{trunc}(x . y): \operatorname{trunc}(a) \leq \operatorname{trunc}(x . y) \leq \operatorname{trunc}(b)\}, \max \{\operatorname{trunc}(x . y):$ $\operatorname{trunc}(a) \leq \operatorname{trunc}(x . y) \leq \operatorname{trunc}(b)\}]-$ by monotonicity $-=[\operatorname{trunc}(a), \operatorname{trunc}(b)]$

(3) $C I R$ (trunc) is monotone: If $[a, b] \sqsubseteq[c, d]$, then $a \leq c \leq d \leq b$, and (by monotonicity of trunc) $\operatorname{trunc}(a) \leq \operatorname{trunc}(c) \leq \operatorname{trunc}(d) \leq \operatorname{trunc}(b)$, therefore $C I R(\operatorname{trunc})[a, b]=[\operatorname{trunc}(a), \operatorname{trunc}(b)] \sqsubseteq[\operatorname{trunc}(c), \operatorname{trunc}(d)]=C I R(\operatorname{trunc})$.

Note that, although trunc is not a continuous function according to euclidean topology, and that some computability models for real numbers accept just continuous function (e.g. Type-2 machines [9]), it is intuitively a computable function in systems which work with representations for real numbers. Note also that, in some

\footnotetext{
${ }^{18}$ Note that, in the Scott sense, $\bigcap \Delta=\sqcup \Delta$.
} 
other models of computability, e.g. real-RAM [6], the trunc function is computable and it is implementable in some concrete machine.

Proposition 4.1. CIR(trunc) is nor Moore-continuous neither Scott-continuous.

Moore and Scott continuity does not capture some computable functions like trunc ${ }^{19}$. It seems that the preservation of least upper bounds by Scott continuity is something too strong, therefore it is necessary to find some definition between monotonicy and ord-continuity to capture functions like trunc.

\section{Final remarks}

Some real functions have many different analytic expressions; for example: $f(x)=$ $2 x$ can be expressed by $(1+1) x, x+x$, etc. Using a real arithmetic formula (i.e. an expression containing variables, real numbers, and operations) for $f$, replacing real variables occurrences by the corresponding interval variables and real arithmetic operations by the corresponding interval operations, the resulting expression designates an interval function which is called natural extension for $f$ (see Moore [5] p. 21). However, the natural extensions of those expressions are different interval representations of $f$, which contain $C I R(f)^{20}$. In some cases, there are expressions in which the extension coincides with $C I R(f)$; for example: The function above, $f(x)=2 x$, is such that $C I R(f)([a, b])=[\min f([a, b]), \max f([a, b])]=[2 a, 2 b]=$ $[2,2] *[a, b]$, which is the natural extension of the expression $2 x$. In this case we have an interval expression which denotes $C I R(f)$. However, it is not always possible to find an interval expression which characterizes $C I R(f)$; for example: Given the function $f(x)=x^{2}$, its $C I R(f)[a, b]=\left[\min \left\{x^{2}: x \in[a, b]\right\}, \max \left\{x^{2}: x \in[a, b]\right\}\right]$, is rewritten as:

$$
C I R(f)[a, b]= \begin{cases}{\left[a^{2}, b^{2}\right],} & \text { if } a \geq 0 \\ {\left[b^{2}, a^{2}\right],} & \text { if } b<0 \\ {\left[0, \max \left\{a^{2}, b^{2}\right\}\right],} & \text { otherwise. }\end{cases}
$$

Clearly, there is no arithmetical expression which describes this function. Therefore it is not a natural extension of any real arithmetical expression.

Although natural extensions are very easy to obtain and are clearly more tractable from the computational point of view, they are not broad enough to describe any real continuous function - e.g. there does not exist the natural extension for $\sin (x)$ although it is possible to find an algorithm to compute $C I R(\sin )$. Finally they do not always describe the best interval representation for $f-C I R(f)$.

All the notions and proofs of this section are trivially extended for $\mathbb{R}^{n}$ and $\mathbb{I}(\mathbb{R})^{n}$.

\footnotetext{
${ }^{19}$ trunc is a kind of accessory function, it is just a change of space; it is a coercion of data-types.

${ }^{20} \mathrm{Or}$ in the Scott theory: is an information for $C I R(f)$.
} 


\section{References}

[1] B.M. Acióly and B.R.C. Bedregal, A quasi-metric topology compatible with inclusion monotonicity on interval space, Reliable Computing, 3, No. 3 (1997), 305-313.

[2] B.M. Acióly, "Computational foundations of interval mathematics", Ph.D. thesis, in Portuguese, Instituto de informática, Universidade Federal do Rio Grande do Sul, Dezembro 1991.

[3] E. Loh and G.W. Walster, Rump's example revisited, Reliable Computing, 8, No. 3 (2002), 245-248.

[4] A. McCluskey and B. McMaster, "Topology Course Lecture Notes", Topology Atlas, 1999. Available in http://at.yorku.ca/i/a/a/b/23.htm.

[5] R.E. Moore, "Methods and applications of interval analysis", SIAM Studies in Applied and Numerical Methematics, SIAM, 1979.

[6] E. Novak, The real number model in numerical analysis, Journal of Complexity, 11, No. 1 (1995), 57-73.

[7] D. S. Scott, Outline of a mathematical theory of computation, in "4th Princeton Conference on Information Science and Systems", pp. 65-106, 1970.

[8] J. Stoy, "Denotational semantics: The Scott-Strachey approach to programming language theory", MIT Press, Massachusetts, 1977.

[9] K. Weihrauch, "Computable analysis - an introduction", Springer Verlag, 1997. 